\title{
A Circuit-Model-Navigated Design Process and Efficiency Estimation for Short-Circuited Self-Excited EBG Resonator Antenna
}

\author{
Mehdi Hosseini \\ The Edward S. Rogers Sr. Department of Electrical \& Computer Engineering, 10 King's College Road, Toronto, ON, Canada M5S $3 G 4$ \\ Correspondence should be addressed to Mehdi Hosseini; mehdi.hosseini@utoronto.ca \\ Received 25 February 2016; Revised 1 May 2016; Accepted 10 May 2016 \\ Academic Editor: Ikmo Park \\ Copyright (C) 2016 Mehdi Hosseini. This is an open access article distributed under the Creative Commons Attribution License, \\ which permits unrestricted use, distribution, and reproduction in any medium, provided the original work is properly cited.

\begin{abstract}
The paper presents a circuit model for the computationally efficient design of a planar Short-Circuited Self-Excited EBG Resonator Antenna (SC SE-EBG-RA). To this purpose, the same circuit model previously presented for the Open-Circuited version of the antenna is modified to be applicable to the SC version. Detailed HFSS modeling and simulation corroborate the accuracy of the model in predicting the antenna resonance. The efficiency of the designed antenna is calculated by a simulated Wheeler Cap Method (WCM) and is compared with the standard efficiency given by the numerical analyzer. The EM modeling is arranged so as to incorporate the effects of the SMA connector, discontinuities, and the WC, emulating a real WC measurement and yielding a high degree of confidence in the results. Overall, a small antenna sized $0.34 \lambda \times 0.30 \lambda \times 0.03 \lambda$ with $93 \%$ verified efficiency is achieved, which is also compiled with affordable manufacturing processes.
\end{abstract}

\section{Introduction}

Although electrically small antennas (ESAs) have been under study for years [1], it was the recent boom in modern communications systems which truly revealed their importance. On the bright side of this evolution, the gradual shift over to higher frequencies has brought the opportunity of using shorter wavelengths, thus employing physically smaller antennas. However, the insatiable data demand by users continues to put ever-increasing pressure on the design of ESAs. The proliferation of frequency bands and the desire to incorporate as many allocated bands as possible in contemporary design scenarios have also led to the situation. The main challenge in achieving a desirable ESA is the fundamental $[1,2]$ compromise between bandwidth (BW), gain, and the electrical size of the antenna. When attempting to reduce the electrical size, the typical cost is to have a reduced $\mathrm{BW}$ or deteriorated radiation efficiency, or even both, a fact which turns the antenna miniaturization into an art of compromise [3]. One of the main avenues of research, related to ESAs, is to develop computationally efficient analysis methods for the design of such EM structures, which mainly involves developing quasistatic circuit models. Circuit models provide the designer with a set of initial values for time-consuming design optimization processes and, by doing that, streamline the course of achieving the most optimized ESA, even under stringent requirements. Such models can also provide one with a rapid insight into the relationships between performance and structural parameters [4], spurring more innovation and better problem-solving ideas in antenna design. In case the antenna possesses a periodic structure [5-8], first, the unit cell is modeled separately $[9,10]$, and then the acquired knowledge is fed to the theories such as Bloch $[7,11,12]$, to understand the performance of the whole periodic antenna composed of the unit cells.

One of the most recent examples of periodic ESAs, for which a Bloch-based circuit model is proposed, is the socalled Self-Excited EBG Resonator Antenna (SE-EBG-RA) $[4,8,13,14]$. The SE-EBG-RA was first introduced in [7] as a new type of high-efficiency planar ESA, the principle of operation of which was explained using a Bloch diagram. Most typical EBG antennas are based on an antenna such as dipole [15, 16], patch [17], or other variations [18], placed over a high impedance plane, composed of various kinds of unit 
cells (e.g., [19]). However, the feature which differentiates the SE-EBG-RA from most of such conventional EBG antennas is its special integrated configuration and radiation mechanism. Few cascaded EBG cells are viewed as a piece of a highly radiating TL, which is directly excited (Self-Excited) by a microstripline (MSL) on one side, while the other side could be open [4, 7], shorted [13], or even terminated [8]. This way, the radiator and the EBG cells form one unified radiating structure. Despite such differences in mechanism and structure, the performance of this type of periodic antenna is quite similar to other EBG antennas. Following these efforts, [4] presented a relatively accurate Bloch-based circuit model for rapid analysis and design of SE-EBG-RAs, while the model was only applicable when the cells were left open on one side (Open-Circuited), forming an OC SE-EBGRA. Also, the equations were only valid for three unit cells, while not a necessity, as demonstrated later in [13].

To supplement the model in [4], this work presents a new generalized version which is applicable not only to the OC SE-EBG-RA but also to the SC version with any number of cells (Section 2). In Section 3, a sample SC SE-EBG-RA is designed through an efficient design process, navigated by the proposed circuit model. Next, in Section 4, a Wheeler Cap (WC) is modeled along with the designed antenna to numerically estimate the efficiency of the antenna. In-detail numerical modeling and analyses provide two independent estimations of $\eta$. Afterwards, an alternative versification of the EM model is rendered in Section 5, and, then, the paper is concluded by Section 6 in which the effects of extreme truncation of the antenna ground plane on its performance are investigated. All numerical analyses are carried out using the High Frequency Structure Simulator (HFSS).

\section{Circuit Model for the SC SE-EBG-RA}

The SE-EBG-RA is composed of small periodic metal patches, deployed on a grounded substrate, with tiny gaps in between. The dispersion relation of this periodic structure, considered as a type of periodic TL, is [4]

$$
\beta^{2}=k^{2}-\frac{\sqrt{\varepsilon_{\mathrm{reff}}}}{Z_{0} d C_{g} c},
$$

where $k$ is the wavenumber [4] of the unloaded MSL (with gaps removed), $c$ is $\left(\varepsilon_{0} \mu_{0}\right)^{-1 / 2}$, and $d$ is the cell size which must be much smaller than free-space wavelength, for validity of (1). $Z_{0}$ and $\varepsilon_{\text {reff }}$ are also found from equations in [4]. The cutoff frequency $\left(f_{c}\right)$ of the dispersion diagram is expressed by [4]

$$
\omega_{c}^{2}=\frac{c}{Z_{0} d C_{g} \sqrt{\varepsilon_{\text {reff }}}}
$$

where $C_{g}$ is the capacitance caused by coplanar coupling $\left(C_{c p}\right)$ between the adjacent patches. The parallel plate coupling considered in [4] is neglected in this work as traces are thin. If $d$ is much larger than the gap size, as it is in Figure $1(\mathrm{c}), C_{\mathrm{cp}}$ can be calculated from $C_{g}=C_{\mathrm{cp}}=2 C_{\mathrm{cp} 1}+$ $C_{\mathrm{cp} 2}$ (see Figure 1), the equations of which are given in [4].
The resonance frequency $\left(f_{r}\right)$ of the $m$-cell OC SE-EBG-RA can be expressed by

$$
\omega_{r}^{2}=\omega_{c}^{2}+\omega_{p}^{2}
$$

where $\omega_{r}$ is $2 \pi f_{r}$ and $\omega_{p}$ is

$$
\omega_{p}=\frac{c \pi}{n l \sqrt{\varepsilon_{\mathrm{reff}}}},
$$

where, for the OC case in [4], $n=2$. Also, $l=m d$, where $m$ is the number of cells. Possibility of using different numbers of cells for SE-EBG-RA was demonstrated in [13]. It was also shown there that the SC version is naturally a quarterwavelength antenna, as opposed to the OC version which is half-wavelength. Thus, rather than $\beta l=\pi / 2$ used in [4], we can insert $\beta l=\pi / 4$ into (1) and find $f_{r}$ of the SC version in a similar manner. Manipulation of the equations suggests that (1) to (4) can be reused, provided that $n=4$ rather than 2 for the OC case. It is noted that, for Figure 1 with 2 cells, $m=2$. Equations (1) to (4) provide the designer with a new set of relatively accurate closed-form equations which streamline the design process of the 2-cell SC SE-EBG-RA in Figure 1.

It is noted that (4) and the clarifications above are specifically presented for the SC version (not included in [4]) and are presented in this work for the first time, although looking similar to equations in [4]. Moreover, the present effort supplements and generalizes the circuit model in [4], as the previous model was applicable to the OC version with only three cells $(m=3)$. In contrast, in this version, $m$ can be arbitrarily chosen, within the limits described in [13].

\section{Circuit-Model-Based Design Process}

If we apply applying the proposed model to Figure 1(a), while considering the structural parameters in Figure 1(c), $f_{r}=$ $5.53 \mathrm{GHz}$. This $f_{r}$ is closely corresponding $(\Delta \sim 3 \%)$ to the HFSS-simulated $f_{r}$ of $5.37 \mathrm{GHz}$, demonstrating the accuracy of the circuit model. This accuracy was also observed in [4] for the OC antenna version.

In practice, however, it is easier to realize the shorting plate of Figure 1(a) with vias. Therefore, as in Figure 1(b), the plate is replaced with two vias. Table 1 compares the antenna properties obtained from the model and from the HFSS simulation for the 2-via and plate versions. As seen, replacing the plate with vias has caused $\sim 19 \%$ increase in $f_{r}$ to $f_{r}=6.38 \mathrm{GHz}$, at which $\eta_{\text {HFSS }}$ is $85 \%$. Figure 2 also shows the radiation pattern of the 2 -via version and demonstrates that the pattern is broadside with very little back radiation.

The $19 \%$ shift is quite expected as the two vias cannot exactly emulate the same effects of the shorting plate, and hence, the antenna retains some of its OC characteristics around the vias, resulting in a frequency shift. Despite this shift, the design is deliberately based on vias to make it compatible with the standard PCB process. In addition, the adoption of $\mathrm{RO} 4350 \mathrm{~B}\left(\varepsilon_{r}=3.66 ; \tan \delta=0.004\right)$ is another effort to meet the requirements of $\mathrm{PCB}$ manufacturing because this substrate is regarded as the low-loss pair of the FR4 and thus is demanded by volume-manufacturers. It 


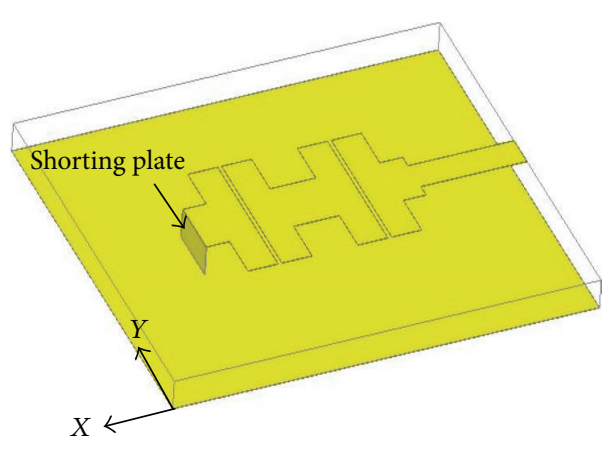

(a)

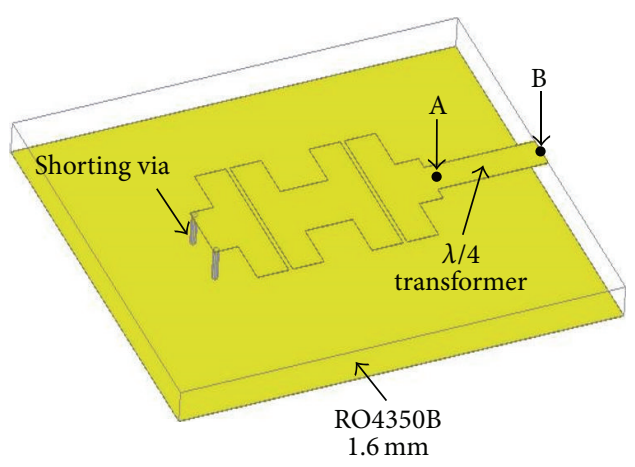

(b)

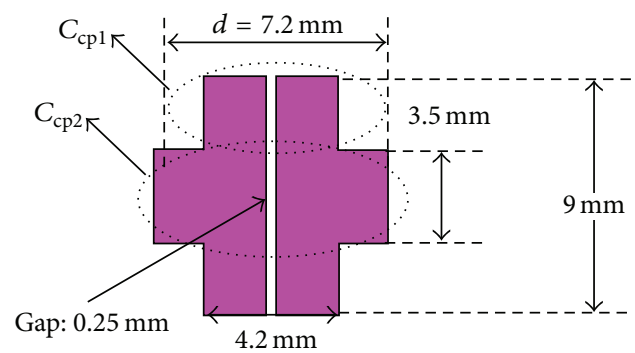

(c)

FIGURE 1: SC SE-EBG-RA composed of two unit cells: (a) with a shorting plate and (b) with vias acting as shorting plate; traces are $30 \mu \mathrm{m}$ copper; the $\lambda / 4$ transformer is $2.45 \mathrm{~mm}$ wide and $6.96 \mathrm{~mm}$ long; the via radius is $0.25 \mathrm{~mm} ; 28 \mathrm{~mm} \times 24 \mathrm{~mm}(X Y)$; substrate is $1.6 \mathrm{~mm}$ thick RO4350B.

TABLE 1: HFSS-simulated properties of the antennas in Figure 1 as compared with the circuit model results; $\Delta$ is the change in $f_{r}$, divided by $f_{r}$ given by the model; $\lambda$ is calculated at $f_{r}$.

\begin{tabular}{lcccc}
\hline SC SE-EBG-RA with 2 unit cells & $f_{r}(\mathrm{GHz})$ & $\eta_{\text {HFSS }}(\%)$ & $\Delta f_{r}(\%)$ & $X Y Z$-dimensions $(\lambda)$ \\
\hline Model & 5.53 & - & 0 & $0.52 \times 0.44 \times 0.029$ \\
Plate & 5.37 & 83.5 & 2.9 & $0.50 \times 0.43 \times 0.029$ \\
2 vias & 6.38 & 85 & 15.37 & $0.60 \times 0.51 \times 0.034$ \\
\hline
\end{tabular}

should be noted that a thick-metal version of the antenna in Figure 1(a), with high-aspect-ratio gaps on a high dielectric substrate, was introduced for the first time in [13], referred to as the SC SE-EBG-RA. On the contrary, the proposed design is thin-trace (similar to antenna versions in $[14,20]$ ), replaces the shorting plate with vias (ease of fabrication), is based on a desirable low dielectric material, and maintains the relatively high efficiency reported in [13]. Figure 3 renders a plot of the input impedance of the antenna in Figure 1(b), before (see point A) and after (see point B) the application of the transformer. As seen, the behavior at point A matches a parallel RLC model, and the transformer has changed the model to series one around the operating frequency of $6.38 \mathrm{GHz}$.

\section{Application of WCM to the SC SE-EBG-RA}

In this section, a rectangular WC is meticulously modeled in HFSS and placed over the antenna designed in Section 3. The design in Figure 1(b) has XY-dimensions of $28 \times 24$ (in $\mathrm{mm})$; thus, the cap will adopt the same lateral dimensions. When choosing the cap height, however, some important considerations are necessary. Fundamentally, in Wheeler Cap Method (WCM), the cap dimensions must be electrically small to prevent the excitation of resonant modes in the cap, which could potentially act as a rectangular cavity [21]. On the other hand, the cap should be large enough so that no part of the cap could interfere with the antenna near fields, keeping in mind that such fields could extend up to $\sim \lambda / 3$ [22] from the antenna phase center or, roughly speaking, from the antenna physical center. With a cap height of $15 \mathrm{~mm}$, the cap is similar to a rectangular cavity resonator with $X Y Z$ dimensions of $28 \times 24 \times 15$ (in $\mathrm{mm}$ ). The related textbook equation [23] gives the cut-off frequency $\left(f_{c}\right)$ of the dominant mode, $\mathrm{TE}_{101}$, of such a resonator as $11.34 \mathrm{GHz}$, which is favorably far enough from $f_{r}$. It is worth mentioning that, in [13], it was theoretically demonstrated that the SC SEEBG-RA is naturally a quarter-wave shorted resonator, which always follows a parallel RLC model. This feature is also deduced from point A impedance in Figure 3. When applying the transformer, however, as in Figure 3, the impedance will flip over to roughly series RLC one (point B in Figure 1(b)), which justifies the use of the WCM. For this reason, in [13], for a prototyped thick-metal SC SE-EBG-RA, which was 

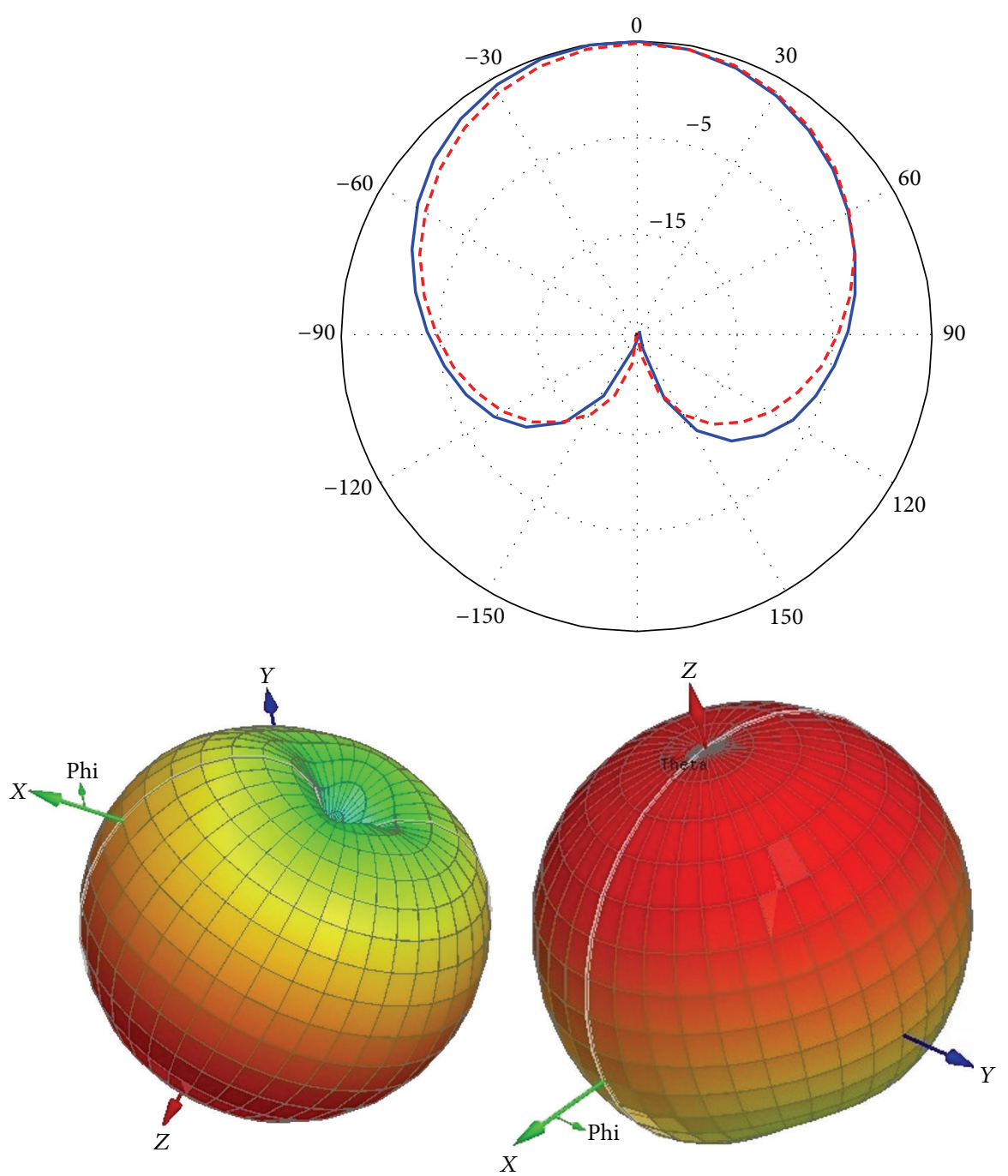

$4.8561 e+000$

$2.2138 e+000$

$-4.2855 e-001$

$-3.0709 e+000$

$-5.7132 e+000$

$-8.3555 e+000$

$-1.0998 e+001$ ర్ర

$-1.3640 e+001$

$-1.6282 e+001$

$-1.8925 e+001$

$-2.1567 e+001$

$-2.4209 e+001$

FIGURE 2: 2D and 3D radiation patterns of the antenna in Figure 1(b) at $6.38 \mathrm{GHz}$; ground plane size is $28 \mathrm{~mm} \times 24 \mathrm{~mm}(X Y)$; solid line is for the E-plane $(X Z)$ and dashed line is for the H-plane; for clarity, two perspectives are provided in $3 \mathrm{D}$ case; the realized gain ( $\mathrm{dBi}$ ) is plotted.

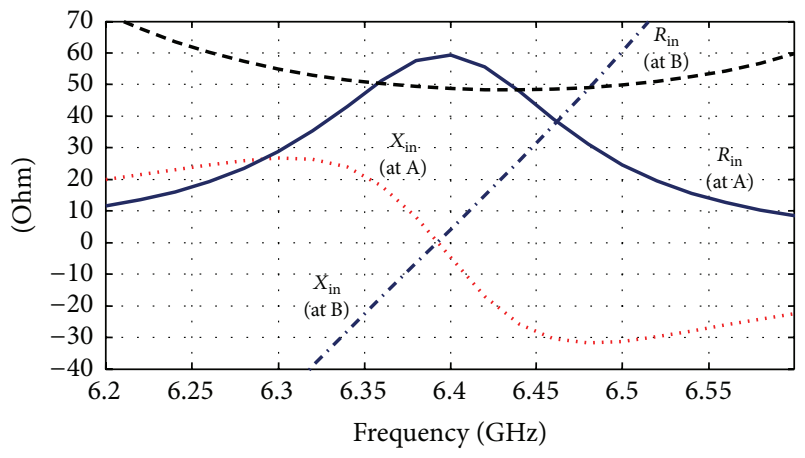

FIGURE 3: HFSS-simulated input impedance of Figure 1(b) at point A (parallel RLC model) and point B (series RLC model).

matched by a $\lambda / 4$ transformer, the actual WCM yielded an accurate estimation of the efficiency.
Figure 4 shows a comprehensive HFSS model which exactly simulates a typical SMA connector, a short piece of coaxial cable, the cap (Figure 4(b)), and the antenna in Figure 1(b). The connector and cable are modeled with inner and outer conductor diameters of $0.5 \mathrm{~mm}$ and $2.4 \mathrm{~mm}$, respectively. Considering $\varepsilon_{r}=3.1$, as with some commercial connectors, and applying the related equation, $Z_{0}=$ $\left(60 / 3.1^{1 / 2}\right) \ln (2.4 / 0.5)$ [23], $53.45 \Omega$ is obtained. A waveport is placed at the beginning of the coaxial cable to excite the structure for which the HFSS-calculated $Z_{0}$ is satisfactorily $53.50 \Omega$. In Figure $4(\mathrm{c})$, the input impedance $\left(Z_{\text {in }}=R_{\text {in }}+\right.$ $j X_{\text {in }}$ ) is calculated at the beginning of $\lambda / 4$ transformer (point A in Figure 4(a)); that is to say, the effects of the connector and the cable are modeled and then deembedded. As a result, $f_{r}$ slightly shift from $6.38 \mathrm{GHz}$ in Figure 1(b) to $6.47 \mathrm{GHz}$ in Figure $4(\mathrm{c})$. This strategy helps emulate what occurs in an actual measurement using WCM. Without the cap, $R_{\text {in }}=50 \Omega$ while when the cap is on, $R_{\text {in }}=10.5 \Omega$. Therefore, $\eta_{\mathrm{wc}}=$ $(50 \Omega-10.5 \Omega) / 50 \Omega=79 \%$. However, when the cap is placed, 


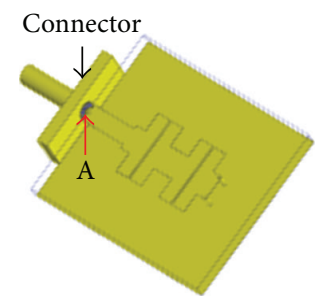

(a)

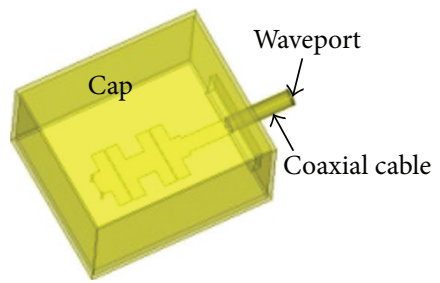

(b)

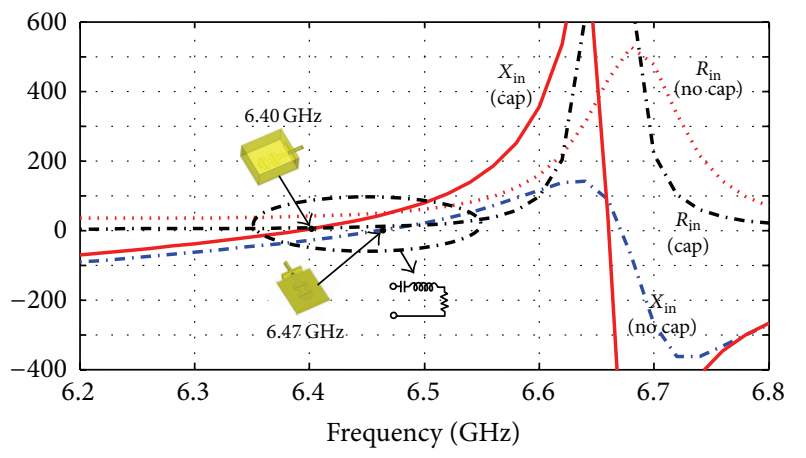

(c)
Figure 4: Detailed EM model of (a) the antenna and (b) the WC on the antenna, along with (c) the simulated $Z_{\text {in }}(\Omega)$ of (a), with and without the WC; the WC electrical dimensions are $\sim 0.60 \lambda \times$ $0.52 \lambda \times 0.32 \lambda$; connector and cable dimensions are given in the text. Connector housing details are as in Figure 5.

$f_{r}$ is no longer $6.47 \mathrm{GHz}$ and slightly drops to $6.40 \mathrm{GHz}$. Thus, one can alternatively read $R_{\text {in }}$ at this new frequency, which is $6.5 \Omega$, resulting in $\eta_{\mathrm{wc}}=87 \%$. Because this frequency shift is as low as $\sim 1 \%$, it is more reasonable to take the average between $6.5 \Omega$ and $10.5 \Omega(8.5 \Omega)$ and use it along with the WCM. In this case, $\eta_{\text {wc }}=(50 \Omega-8.5 \Omega) / 50 \Omega=83 \%$, which is only $2.5 \%$ lower than $85 \%$ ( $\left.\eta_{\mathrm{HFSS}}\right)$.

$\eta_{\text {HFSS }}$ is independently calculated by the HFSS built-in method which, according to the HFSS manual, is the ratio of the radiated power (integrated over the radiation box circumscribing the structure) to the input real power. It is worth noting here that, in [13], the measured $\eta_{\mathrm{wc}}$ of an OC SE-EBG-RA prototype was shown to be similarly lower than $\eta_{\mathrm{HFSS}}$ by $7 \%$. This difference was attributed to the well-known observation that efficiencies calculated by HFSS are usually somewhat higher than reality.

\section{An Alternative Verification of the EM Model}

One more consideration which lends more credibility to the simulation results in Figure 4 and obviates the need for prototyping is to numerically evaluate the cable and SMA connectors, without the antenna. Figure 5 shows an EM model in HFSS which exactly models a 2-port network including the connector, cable, and a fraction of $50 \Omega$ MSL. As in Figure 6, the corresponding $S$-parameters confirm that the network operates satisfactorily, which means almost all the power, as expected, is delivered from port 1 to port 2 . This technique assures one of the accuracies of the excitation of

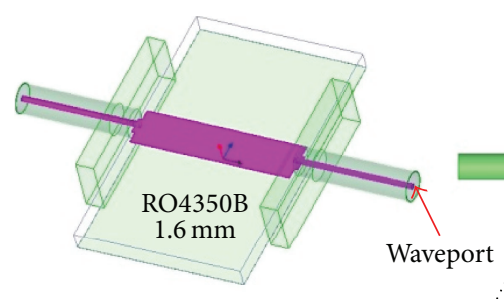

$3 \mathrm{D}$ view
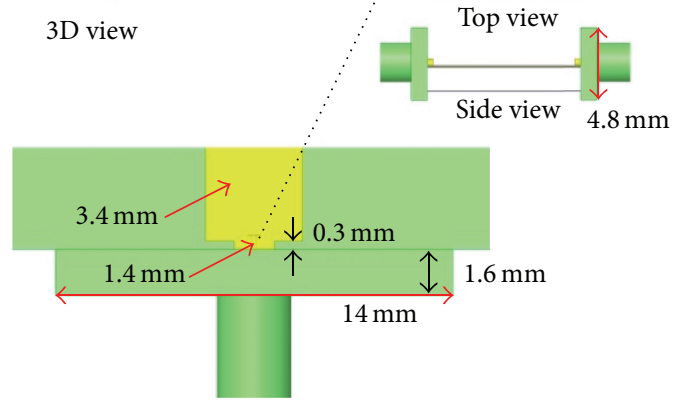

FIGURE 5: Different view of a detailed EM model, including the MSL, SMA connector, and pieces of coaxial cable. Inner/outer dimensions of the connector and cable are given in the text.

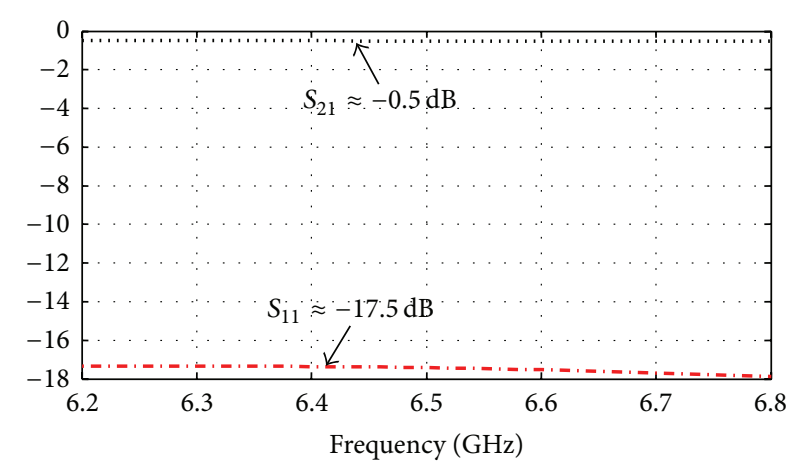

Figure 6: Simulated $\left|S_{11}\right|(\mathrm{dB})$ and $\left|S_{21}\right|(\mathrm{dB})$ associated with Figure 5; effects of discontinuities and SMA connector are included.

waves at ports and reliable modeling of interconnects and, therefore (from a new standpoint), verifies the EM model in Figure 4.

It is worth mentioning that the verification approach in this paper is entirely simulation-based. In Section 3, it is shown that the resonance frequencies calculated by HFSS and the circuit model agree well $(\Delta<2.9 \%)$. Moreover, in Section 4 , it is demonstrated that the efficiencies calculated by HFSS and the simulated-WC method correspond well $(\Delta<$ $2.5 \%)$. The analysis in this section is also a third effort for verification of antenna feeding parts. Such simulated-based approaches are applicable to small antennas, considering the small electrical size of the structures. In this case, the whole structure, including the WC, is only $0.60 \lambda \times 0.52 \lambda \times 0.32 \lambda$. While the simulation of such small EM models can be readily handled using inexpensive computers, in case the model is large (e.g., array antennas), prototyping and measurement are inevitable. 
TABLE 2: The effect of truncation on the SC SE-EBG-RA (2-via); $\Delta$ is the change in $f_{r}$, divided by $f_{r}$ of the nontruncated case; the connector and cable are exactly modeled in both cases; $G_{r}$ stands for the realized gain.

\begin{tabular}{|c|c|c|c|c|c|c|}
\hline & $f_{r}(\mathrm{GHz})$ & $D_{\text {HFSS }}(\mathrm{dBi})$ & $G_{r}(\mathrm{dBi})$ & $\eta_{\text {HFSS }}(\%)$ & $\Delta f_{r}(\%)$ & $X Y Z$-dimensions $(\mathrm{mm})$ \\
\hline Nontruncated (Figure 4(a)) & 6.47 & 6.15 & 5.42 & 84.5 & 0 & $28 \times 24 \times 1.6(0.60 \lambda \times 0.52 \lambda \times 0.035 \lambda)$ \\
\hline Truncated (Figure 7) & 6.36 & 4.83 & 4.37 & 93 & 1.7 & $16 \times 14 \times 1.6(0.34 \lambda \times 0.30 \lambda \times 0.03 \lambda)$ \\
\hline
\end{tabular}

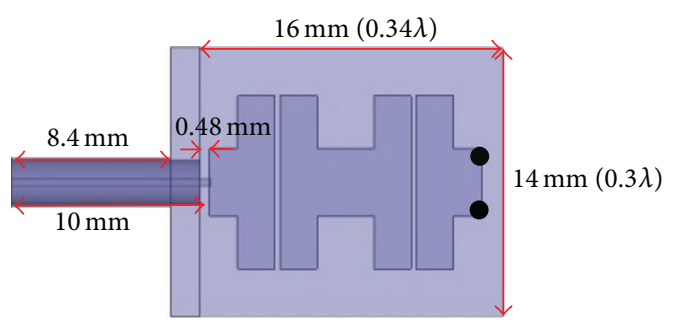

Figure 7: Truncated version of the SC SE-EBG-RA in Figure 1(b) including the SMA and the connectors; unit cell dimensions are as in Figure 1(c).

\section{Extremely Truncated SC SE-EBG-RA}

The last study is to investigate the sensitivity of the SC SEEBG-RA in Figure 4(a) against truncation and the possibility of further miniaturization obtained through reducing the ground plane size. As in Figure 7, to save the footprint, the $\lambda / 4$ transformer is removed as the edge impedance at point $\mathrm{A}$ of Figure 4 (a) was $\sim 60 \Omega$ (close to $50 \Omega$ ). The ground plane is also truncated to $16 \mathrm{~mm} \times 14 \mathrm{~mm}$. The structural details are shown in Figure 7. As seen, again, a piece of coaxial cable and the SMA are included in the simulations.

Table 2 compares the properties of the truncated version and the original antenna in Figure 4(a). It is interestingly observed that $\eta$ has improved and the antenna electrical size has dropped significantly. Another observation is that although the antenna has undergone a severe truncation, the shift in resonance is only $1.7 \%$.

As expected, the realized gain has dropped to $4.37 \mathrm{dBi}$ (due to a smaller electrical footprint); nonetheless, it is still a significant gain for such a small electrical footprint. Also, the corresponding radiation pattern is shown in Figure 8, demonstrating a reasonable stability of the pattern against truncation (notice the slight movement of the peak towards the connector side). The good broadside pattern implies that the parasitic effects of the connector and the cable are insignificant. Moreover, Figure 9 compares $\left|S_{11}\right|$ (dB) with frequency for the truncated and original versions, showing that the matching quality is maintained, with $73 \%$ enhancement in the BW from $1.9 \%$ to $3.3 \%$.

\section{Conclusion}

The paper introduces a fast and accurate design process for a small antenna, which is navigated by a proposed circuit model. The so-called Short-Circuited SE-EBG-RA is first designed by the model and then undergoes a demonstrative

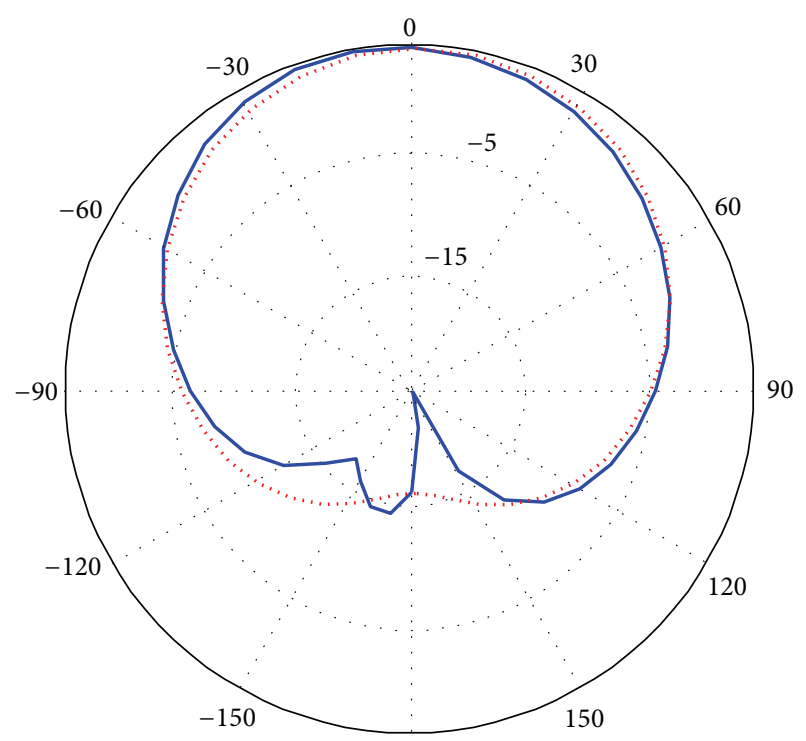

FIGURE 8: Radiation pattern of the antenna in Figure 7 at $6.36 \mathrm{GHz}$; solid line is for the E-plane $(X Z)$ and dashed line is for the H-plane; the realized gain $(\mathrm{dBi})$ is plotted.

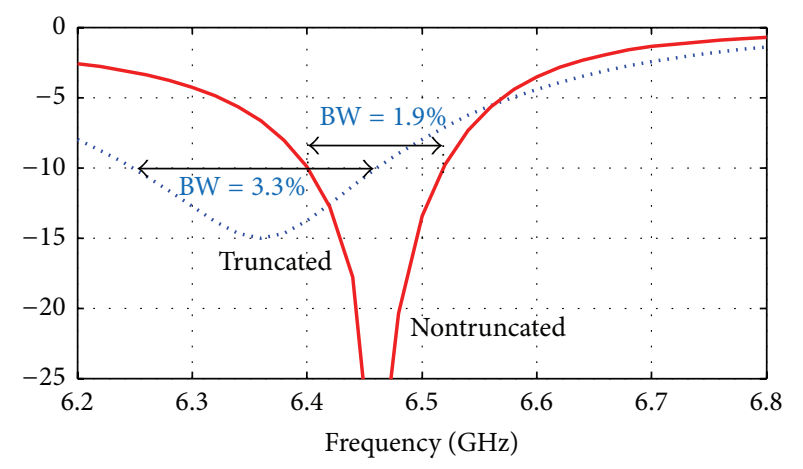

FIGURE 9: Simulated $\left|S_{11}\right|(\mathrm{dB})$, calculated at the waveport of the coaxial cable; truncated antenna in Figure 7 is compared with the nontruncated version in Figure $4(\mathrm{a})$; there is $\sim 1.8 \%$ drop in resonance, owing to truncation.

simulation-based Wheeler Cap (WC) measurement. Comparison between the efficiency calculated by the WC method and the HFSS built-in method favorably shows that there is only $2.5 \%$ difference in estimation. Although the study is entirely based on numerical analysis, in-detail modeling of the connector and the WC yields sufficient confidence in the credibility. The final antenna design proves to be quite robust against truncation of the ground plane, renders a good broadside pattern, and is as small as $0.34 \lambda \times 0.30 \lambda \times$ $0.03 \lambda$. It also exhibits $93 \%$ efficiency, $4.37 \mathrm{dBi}$ realized gain, 
and a respectable $\mathrm{BW}$ of $3.3 \%$. To make the antenna under study applicable to sub- $6 \mathrm{GHz}$ commercial applications, it is designed to operate close to $5.8 \mathrm{GHz}$ unlicensed band and comply with standard PCB process.

\section{Competing Interests}

The author declares that they have no competing interests.

\section{References}

[1] H. A. Wheeler, "Fundamental limitations of small antennas," Proceedings of the IRE, vol. 35, no. 12, pp. 1479-1484, 1947.

[2] W. Geyi, "Physical limitations of antenna," IEEE Transactions on Antennas and Propagation, vol. 51, no. 8, pp. 2116-2123, 2003.

[3] A. K. Skrivervik, J.-F. Zürcher, O. Staub, and J. R. Mosig, "PCS antenna design: the challenge of miniaturization," IEEE Antennas and Propagation Magazine, vol. 43, no. 4, pp. 12-27, 2001.

[4] M. Hosseini, D. M. Klymyshyn, and G. Wells, "A circuit model for the design of self-excited EBG resonator antennas with miniaturized unit cells," IEEE Antennas and Wireless Propagation Letters, vol. 13, pp. 1279-1283, 2014.

[5] J. S. Gomez-Diaz, A. Álvarez-Melcon, and T. Bertuch, "A modalbased iterative circuit model for the analysis of CRLH leakywave antennas comprising periodically loaded PPW," IEEE Transactions on Antennas and Propagation, vol. 59, no. 4, pp. 1101-1112, 2011.

[6] M. Lee, B. A. Kramer, C.-C. Chen, and J. L. Volakis, "Distributed lumped loads and lossy transmission line model for wideband spiral antenna miniaturization and characterization," IEEE Transactions on Antennas and Propagation, vol. 55, no. 10, pp. 2671-2678, 2007.

[7] M. Hosseini and D. M. Klymyshyn, "Radiation properties of EBG textured tall transmission lines and applications: a lowprofile self-excited EBG resonator antenna," IEEE Antennas and Wireless Propagation Letters, vol. 11, pp. 276-280, 2012.

[8] M. Hosseini, High-performance periodic antennas with high aspect ratio vertical features and large intercell capacitances for microwave applications [Ph.D. thesis], University of Saskatchewan, Saskatoon, Canada, 2014.

[9] B. Mohajer-Iravani and O. M. Ramahi, "Wideband circuit model for planar EBG structures," IEEE Transactions on Advanced Packaging, vol. 33, no. 1, pp. 169-179, 2010.

[10] G. Luo, E. Li, X. Wei, and C. Xiang, "Modelling of planar EBG structure by using equivalent circuit method," in Proceedings of the IEEE International Symposium on Electromagnetic Compatibility (EMC '15), pp. 573-576, Dresden, Germany, August 2015.

[11] S. Otto, A. Al-Bassam, A. Rennings, K. Solbach, and C. Caloz, "Transversal asymmetry in periodic leaky-wave antennas for bloch impedance and radiation efficiency equalization through broadside," IEEE Transactions on Antennas and Propagation, vol. 62, no. 10, pp. 5037-5054, 2014.

[12] A. Epstein and G. V. Eleftheriades, "Floquet-Bloch analysis of refracting Huygens metasurfaces," Physical Review B, vol. 90, no. 23, Article ID 235127, 2014.

[13] M. Hosseini, D. M. Klymyshyn, G. Wells, and X. Liu, "Short and open circuited EBG resonator antennas: miniaturization with a shorting plate and dielectric loading," IEEE Transactions on Antennas and Propagation, vol. 63, no. 10, pp. 4305-4314, 2015.
[14] M. Hosseini and D. M. Klymyshyn, "Mutual coupling suppression in an array of 2-layer self-excited EBG resonator antennas," in Proceedings of the IEEE International Symposium on Antennas and Propagation \& USNC/URSI National Radio Science Meeting, pp. 418-419, Vancouver, Canada, July 2015.

[15] R. Ikeuchi and A. Hirata, "Dipole antenna above EBG substrate for local SAR reduction," IEEE Antennas and Wireless Propagation Letters, vol. 10, pp. 904-906, 2011.

[16] L. Akhoondzadeh-Asl, P. S. Hall, J. Nourinia, and C. Ghobadi, "Influence of angular stability of EBG structures on low profile dipole antenna performance," in Proceedings of the IEEE International Workshop on Antenna Technology (IWAT'06), pp. 253256, White Plains, NY, USA, March 2006.

[17] A. Ameelia Roseline, K. Malathi, and A. K. Shrivastav, "Enhanced performance of a patch antenna using spiral-shaped electromagnetic bandgap structures for high-speed wireless networks," IET Microwaves, Antennas and Propagation, vol. 5, no. 14, pp. 1750-1755, 2011.

[18] M. Karaaslan, E. Ünal, E. Tetik, K. Delihacıoğlu, F. Karadağ, and F. Dinçer, "Low profile antenna radiation enhancement with novel electromagnetic band gap structures," IET Microwaves, Antennas and Propagation, vol. 7, no. 3, pp. 215-221, 2013.

[19] D. J. Kern, D. H. Werner, A. Monorchio, L. Lanuzza, and M. J. Wilhelm, "The design synthesis of multiband artificial magnetic conductors using high impedance frequency selective surfaces," IEEE Transactions on Antennas and Propagation, vol. 53, no. 1 I, pp. 8-17, 2005.

[20] M. Hosseini and D. M. Klymyshyn, "Properties of small gaploaded patch antenna with fast-wave behaviours," Electronics Letters, vol. 50, no. 18, pp. 1268-1269, 2014.

[21] D. M. Pozar and B. Kaufman, "Comparison of three methods for the measurement of printed antenna efficiency," IEEE Transactions on Antennas and Propagation, vol. 36, no. 1, pp. 136-139, 1988.

[22] H. A. Wheeler, "The radiansphere around a small antenna," Proceedings of the IRE, vol. 47, no. 8, pp. 1325-1331, 1959.

[23] D. M. Pozar, Microwave Engineering, John Wiley \& Sons, 2nd edition, 1998. 




\section{Enfincering}
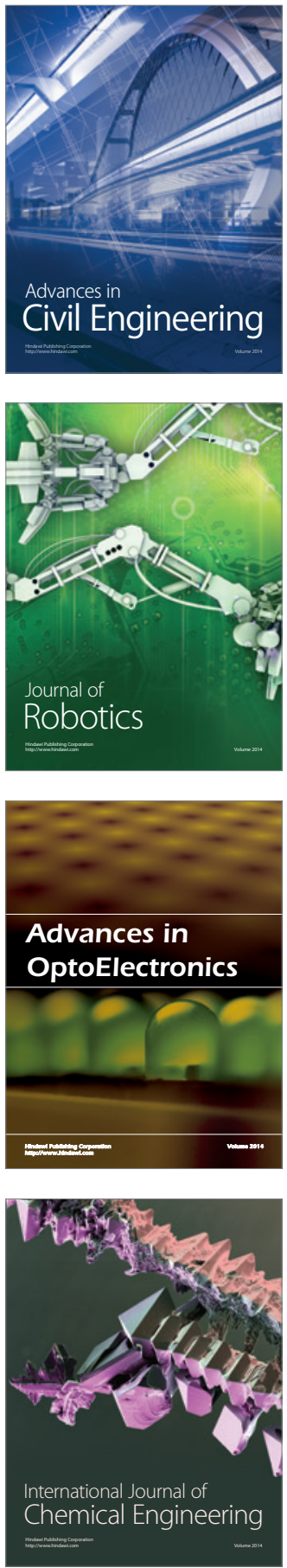

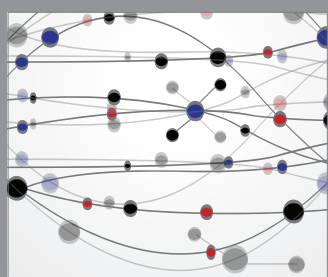

The Scientific World Journal

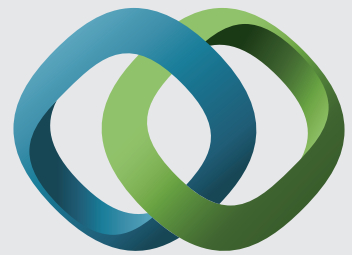

\section{Hindawi}

Submit your manuscripts at

http://www.hindawi.com
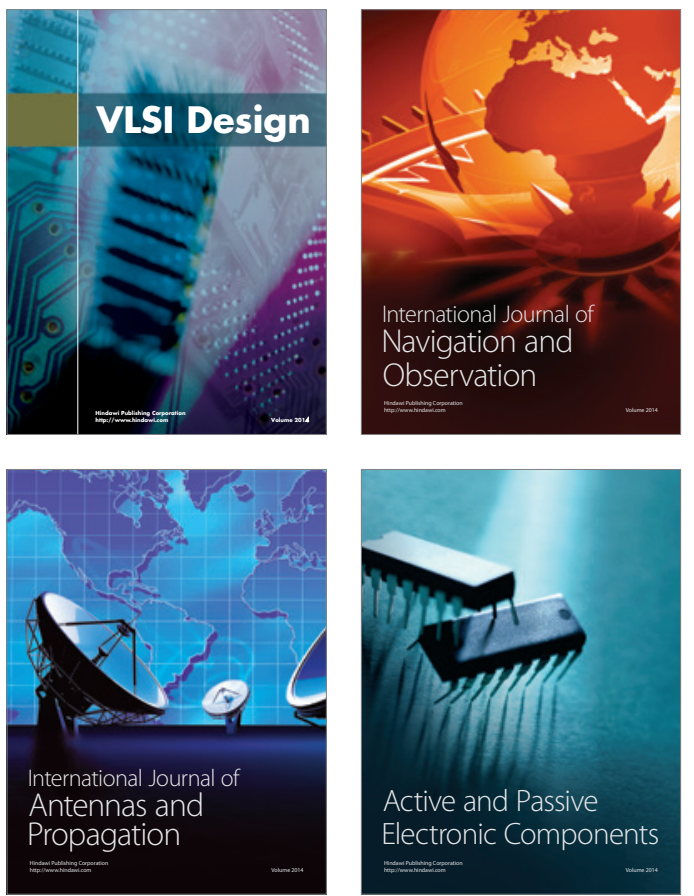
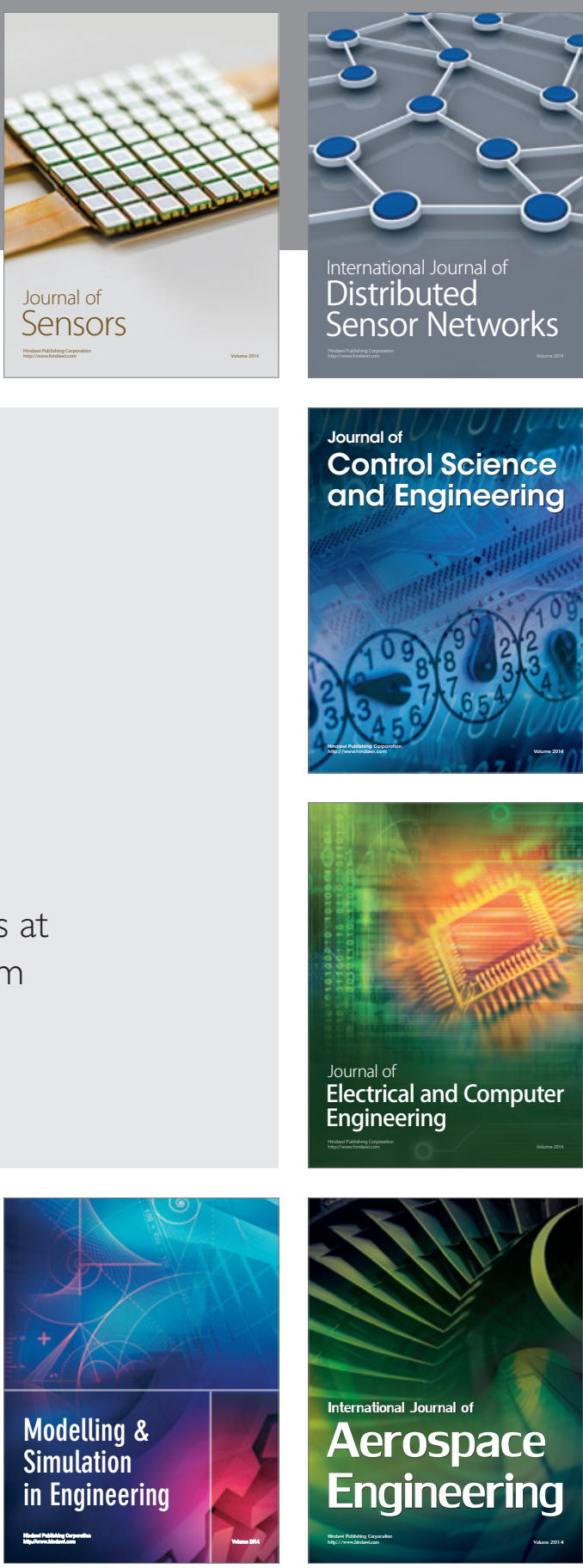

International Journal of

Distributed

Sensor Networks

Journal of

Control Science

and Engineering
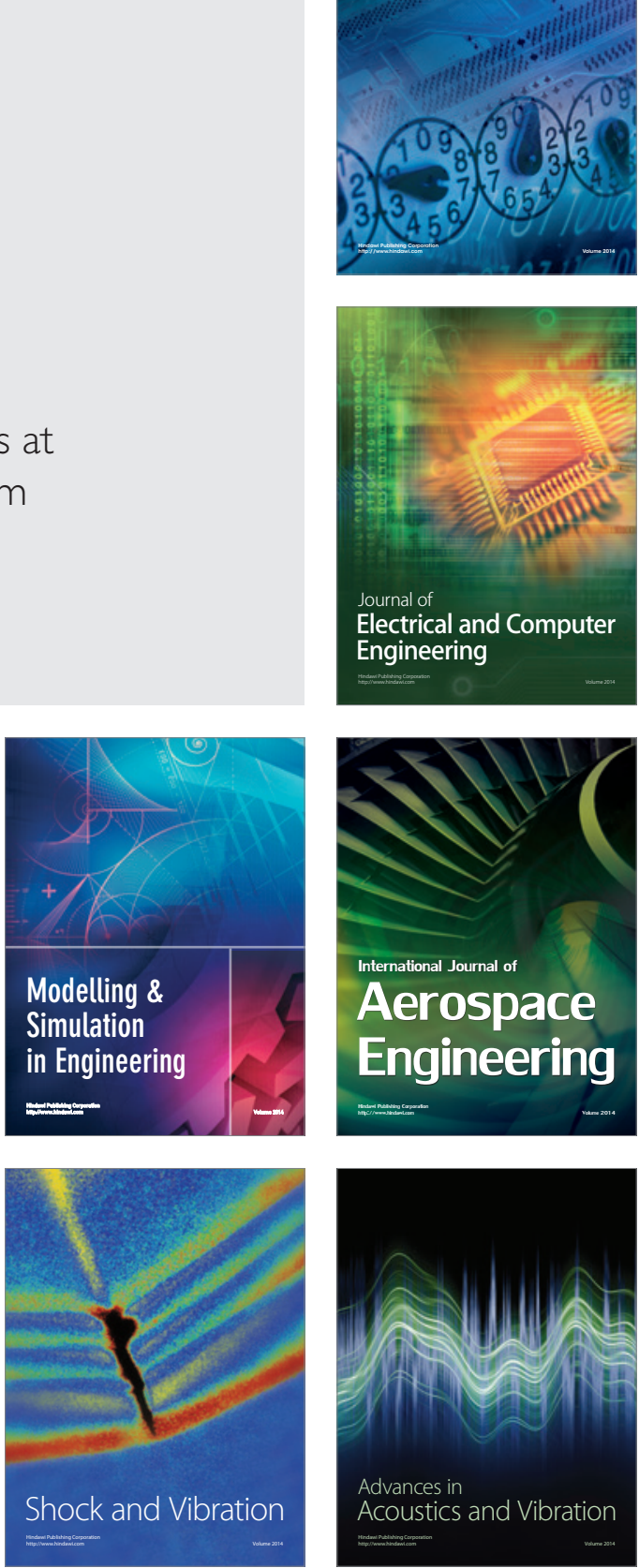